\title{
Novel hydrogels based on yeast chitin-glucan complex: Characterization and safety assessment
}

\author{
Diana Araújo ${ }^{\text {a }}$, Vítor D. Alves ${ }^{\text {b }}$, Sofia A.C. Lima ${ }^{c}$, Salette Reis ${ }^{c}$, Filomena Freitas ${ }^{\text {a,* }}$, Maria A.M. Reis ${ }^{\text {a }}$ \\ a UABIO-REQUIMTE, Departamento de Química, Faculdade de Ciências e Tecnologia, Universidade NOVA de Lisboa, 2829-516 Caparica, Portugal \\ b LEAF, Linking Landscape, Environment, Agriculture and Food, Instituto Superior de Agronomia, Universidade de Lisboa, Tapada da Ajuda, $1349-017$ Lisboa, Portugal \\ c LAQV-REOUIMTE, Departamento de Ciências Ouímicas, Faculdade de Farmácia, Universidade do Porto, Rua de Jorge Viterbo Ferreira, 228, 4050-313 Porto, Portugal
}

\section{A R T I C L E I N F O}

\section{Article history:}

Received 20 June 2019

Received in revised form 7 November 2019

Accepted 18 November 2019

Available online 19 November 2019

\section{Keywords:}

Chitin-glucan complex

Hydrogels

Biocompatibility

\begin{abstract}
A B S T R A C T
Chitin-glucan complex (CGC) was used for the first time for the preparation of hydrogels. Alkali solvent systems, $\mathrm{NaOH}$ and $\mathrm{KOH}$ solutions, either at 1 or $5 \mathrm{~mol} / \mathrm{L}$, were used for CGC dissolution using a freeze-thaw procedure (freezing at $-20^{\circ} \mathrm{C}$ and thawing at room temperature; four cycles). The CGC solutions thus obtained were subjected to dialysis that induced the spontaneous gelation of the biopolymer, yielding translucid hydrogels with a yellowish coloration. Although all CGC hydrogels exhibited porous microstructures, high water content (above 97\%) and good mechanical properties, their morphology, viscoelastic properties and texture were influenced by the type of solvent system used for CGC dissolution, as well as by their ionic strength. The K-based hydrogels presented a less compact network with larger pores and exhibited lower elastic properties. The Na-based hydrogels, on the other hand, exhibited a denser structure with smaller pores and a stiffer gel structure. These results show that it is possible to prepare CGC hydrogels with differing characteristics that can be suitable for different applications. Furthermore, all hydrogels were non-cytotoxic towards L929 fibroblasts and HaCaT keratinocytes. This study demonstrates CGC can be used to prepare biocompatible hydrogels with properties render them promising biomaterials.
\end{abstract}

\section{Introduction}

Hydrogels are three-dimensional macromolecular networks capable of adsorbing high amounts of water, saline and physiological media in their structure and swell [1-3]. These soft and pliable structures can be based on synthetic polymers (e.g. acrylic acid, acrylamide, poly(vinyl alcohol)) or on biopolymers (e.g. gelatin, starch, alginate) [2-4]. The later have attracted considerable interest due to their biocompatibility, biodegradability and tissue-mimicking consistency, and are used in applications that range from agriculture and water treatment, to hygiene products and biomedicine $[1,5]$. Hydrogels can be divided into two types, namely, physical or chemical, depending on the type of procedure used to cross-link their polymer chains. Physical hydrogels are obtained by the establishment of physical interactions (e.g. chain entanglements, van der Waals forces, hydrogen bonds or ionic interactions), which are driven by exposing a polymer solution to specific temperatures, $\mathrm{pH}$ values and/or ionic strength. On the other hand, in chemical hydrogels, chemical cross-linkers (e.g. epichlorohydrin, succinic

- Corresponding author at: Chemistry Department, Campus de Caparica, Faculty of Sciences and Technology, Nova University of Lisbon, 2829-516 Caparica, Portugal,

E-mail address: a4406@fct.unl.pt (F. Freitas). anhydride) are used to induce the formation a hydrophilic network stabilized through covalent linkages $[4,6]$.

Several natural polysaccharides have been used to obtain hydrogels, including starch, alginate, hyaluronic acid, cellulose and chitin. These biopolymers hold great potential due to the high number of hydrophilic functional groups (e.g. hydroxyl groups) in their molecules. Most hydrogels are usually prepared using a procedure that involves polymer dissolution in a suitable solvent system, followed by gelation [1,4]. This constitutes a problem for biopolymers such as cellulose and chitin, whose solubility is limited to toxic and/or corrosive organic solvents (e.g. dimethylacetamide/lithium chloride, tetrabutylammonium chloride/dimethyl sulfoxide). Ionic liquids [6], deep eutectic solvents [7] and alkali or alkali/urea aqueous solutions $[4,8,9]$ have been proposed as alternative solvent systems for the dissolution of cellulose and chitin, and the production of hydrogels.

Chitin-glucan complex (CGC) is a water insoluble, highly hydrophilic biopolymer composed of $\mathrm{N}$-acetylglucosamine and glucose monomers [10]. It is one of the cell-wall components of several yeasts and fungi that confer stability and rigidity to the cell $[11,12]$. CGC is a biocompatible and biodegradable biopolymer that combines antioxidant, antibacterial and anti-inflammatory properties that render it a valuable biomaterial for several applications, including the treatment of obesity and diabetes [13], cosmetic ingredient 
[14], as food additive (GRAS notice No. 412, 2012; http://www.fda. gov), wine clarification [15] and wound healing [16].

Similarly to cellulose and chitin, CGC is insoluble in water and aqueous solutions, and also in most organic solvents. Therefore, the development of CGC into commercial products has been hindered by its intractability. In this study, aqueous alkali systems based on $\mathrm{NaOH}$ or $\mathrm{KOH}$ were used for the first time for CGC dissolution by the freeze/ thaw method, and the resulting solutions were subjected to dialysis to induce the biopolymer's gelation. The CGC hydrogels thus obtained were characterized in terms of morphology, rheology, mechanical properties and biocompatibility.

\section{Materials and methods}

\subsection{Materials}

Yeast biomass was obtained by cultivation of the yeast Komagataella pastoris (DSM 70877) using glycerol as the sole carbon source, as described by Farinha et al. [10]. CGC represented $20 \mathrm{wt} \%$ of the cell dry mass. It was extracted from $K$. pastoris biomass by the hot alkaline procedure described by Araújo et al. [17].

\subsection{Preparation of CGC hydrogels}

Two aqueous alkali solvent systems, namely, $\mathrm{NaOH}$ and $\mathrm{KOH}$, were tested at different concentrations ( $1 \mathrm{~mol} / \mathrm{L}$ and $5 \mathrm{~mol} / \mathrm{L}$ ). CGC powder $(0.5 \mathrm{~g})$ was dispersed in the alkali solvent systems $(25 \mathrm{~g})$ and the suspensions were kept at $-20^{\circ} \mathrm{C}$ for $48 \mathrm{~h}$. During this period, four freezethaw cycles were performed in which the thawed suspensions were extensively stirred (at $500 \mathrm{rpm}$, for $1 \mathrm{~h}$ ), at room temperature. The insoluble fractions of the suspensions were separated from the soluble ones by centrifugation $\left(20,000 \times \mathrm{g}, 30 \mathrm{~min}, 4^{\circ} \mathrm{C}\right)$. CGC hydrogels were prepared by dialysis of the soluble fractions with a 12,000 MWCO membrane (Nadir $囚$, dialysis tubing, Carl Roth) against deionized water for $48 \mathrm{~h}$, at $20^{\circ} \mathrm{C}$, until neutral $\mathrm{pH}$ and constant conductivity values (20 $\mu \mathrm{S} / \mathrm{cm}$ ) were achieved. The hydrogels prepared with $\mathrm{NaOH}$ or $\mathrm{KOH}$ $1 \mathrm{~mol} / \mathrm{L}$ and $5 \mathrm{~mol} / \mathrm{L}$, were labelled as $\mathrm{Na} 1$ and $\mathrm{Na} 5$, or $\mathrm{K} 1$ and $\mathrm{K} 5$, respectively.

\subsection{Chemical characterization of CGC hydrogels}

For quantification of sodium and potassium in the hydrogels, freeze dried CGC hydrogel samples $(\sim 5 \mathrm{mg})$ were hydrolyzed with nitric acid $\left(5 \mathrm{~mL} \mathrm{HNO}_{3} 5 \%, \mathrm{v} / \mathrm{v}\right)$, at $120^{\circ} \mathrm{C}$, for $2 \mathrm{~h}$, under constant stirring. Hydrolyzed samples were filtered $(0.2 \mu$ m nylon, WHATMAN) and their content in sodium and potassium was determined by Inductively Coupled Plasma-Atomic Emission Spectrometry (ICP-AES) (Horiba Jobin-Yvon, France, Ultima, equipped with a $40.68 \mathrm{MHz}$ RF generator, Czerny-Turner monochromator with $1.00 \mathrm{~m}$ (sequential) and autosampler AS500). Nitric acid solution $\left(\mathrm{HNO}_{3} 5 \%, \mathrm{v} / \mathrm{v}\right)$ was used as blank, being subjected to the same hydrolysis procedure as the samples.

The water content of the hydrogels was determined according to Eq. (1) [18]:

Water content $(\%)=\frac{(\text { wet mass }- \text { dry mass })}{\text { wet mass }} \times 100$

where the dry mass ( $\mathrm{g}$ ) was determined by freeze drying a pre-weighed amount of the gels (wet mass, $g$ ).

The chemical composition of the hydrogels was analyzed by high performance liquid chromatography (HPLC) after two acid hydrolysis methods, as described by Araújo et al. [17].

\subsection{Rheological properties}

The rheological behavior of the CGC hydrogels was measured and analyzed using a controlled stress rheometer (HAAKE MARSIII, Thermo scientific) equipped with a plate-plate serrated geometry (diameter $20 \mathrm{~mm}$ ) with a gap of $1 \mathrm{~mm}$. The hydrogels samples were equilibrated at $25 \pm 0.03{ }^{\circ} \mathrm{C}$ for $5 \mathrm{~min}$, after which the viscoelastic properties were evaluated by carrying out frequency sweeps at a constant tension within the linear viscoelastic region, for a frequency range from 0.01 to $1 \mathrm{~Hz}$.

\subsection{Texture profile analysis}

The texture properties of the CGC hydrogels were determined with a texture analyzer TA-XT plus (Stable Micro Systems Ltd., Surrey, UK) equipped with a $50 \mathrm{~N}$ load cell. A double compression cycle was carried out up to $50 \%$ strain of the samples original height at a speed rate of $1 \mathrm{~mm} / \mathrm{s}$ using an aluminum plunger with $60 \mathrm{~mm}$ diameter. A delay period of $5 \mathrm{~s}$ was allowed between the two compression cycles. The maximum tension of the first compression was defined as the hardness ( $\mathrm{Pa}$ ) and the ratio between the second and first compression distances until maximum forces was used to measure the springiness. Cohesiveness was defined as the ratio between the positive force area during the second cycle and that of the first compression cycle. All the measurements were performed in duplicate at room temperature $\left(20 \pm 0.2{ }^{\circ} \mathrm{C}\right)$.

\subsection{Cryo-Scanning Electron Microscopy}

The CGC hydrogels were characterized in terms of their structure and morphology by Cryo-Scanning Electron Microscopy (cryo-SEM). The hydrogels were analyzed with a Tabletop Microscope TM3030 (Hitachi in High Technologies, America) equipped with a sample holder with refrigeration. Samples were observed at low temperature $\left(-4{ }^{\circ} \mathrm{C}\right)$ using a magnification in the range of $150-500 \times$.

\subsection{Cytotoxicity tests}

The cytocompatibility of the CGC hydrogels was evaluated according to the International Standard ISO 10993-5:2009(E) through assessment of metabolic activity (MTT (3-(4,5-dimethylthiazol-2-yl)-2,5-diphenyltetrazolium bromide) tetrazolium reduction assay) in two cell lines, human keratinocytes HaCaT and mouse L929 fibroblasts. The cells were grown in Dulbecco's Modified Eagle Medium (DMEM) media supplemented with $10 \%(\mathrm{v} / \mathrm{v})$ fetal bovine serum and $1 \%(\mathrm{v} / \mathrm{v})$ streptomycin/penicillin, and maintained at $37^{\circ} \mathrm{C}$ in a humidified atmosphere of $5 \%(\mathrm{v} / \mathrm{v}) \mathrm{CO}_{2}$. For the cell viability assay, $5 \times 10^{4}$ cells per well were seeded in 96 well plates and cultured at conditions above described. After $24 \mathrm{~h}$, the medium was removed and $100 \mu \mathrm{L}$ of fresh medium containing serial dilutions, up to $1000 \mu \mathrm{g} / \mathrm{mL}$, of freeze dried CGC hydrogels dissolved in alkaline solvent $(\mathrm{NaOH} 1 \mathrm{~mol} / \mathrm{L}$, at neutral $\mathrm{pH})$ were added. Upon $24 \mathrm{~h}$ of exposure, the supernatant was replaced by the MTT solution $(0.5 \mathrm{mg} / \mathrm{mL})$ and incubated $\left(37^{\circ} \mathrm{C}, 5 \%(\mathrm{v} / \mathrm{v}) \mathrm{CO}_{2}\right)$ for $2 \mathrm{~h}$. During incubation, tetrazolium dye MTT is reduced by dehydrogenase enzymes to insoluble formazan. Following the incubation step, the medium was eliminated and replaced with dimethylsulfoxide to dissolve the formazan crystals accumulated inside living cells. For the quantification of formazan produced, the absorbance was measured at $590 \mathrm{~nm}$ using a Synergy ${ }^{\mathrm{TM}}$ HT Multi-mode microplate reader (BioTek Instruments Inc., Winooski, VT, USA), subtracting the background read at $630 \mathrm{~nm}$. Cells grown in fresh medium without treatment and cells treated with Triton $^{\mathrm{TM}} \mathrm{X}-100(1 \% \mathrm{v} / \mathrm{v})$ were used as positive and negative controls, respectively. Results were expressed as percentage of metabolic activity relatively to the values obtained at $2 \mathrm{~h}$ and considering the metabolic activity of non-treated cells as $100 \%$. 


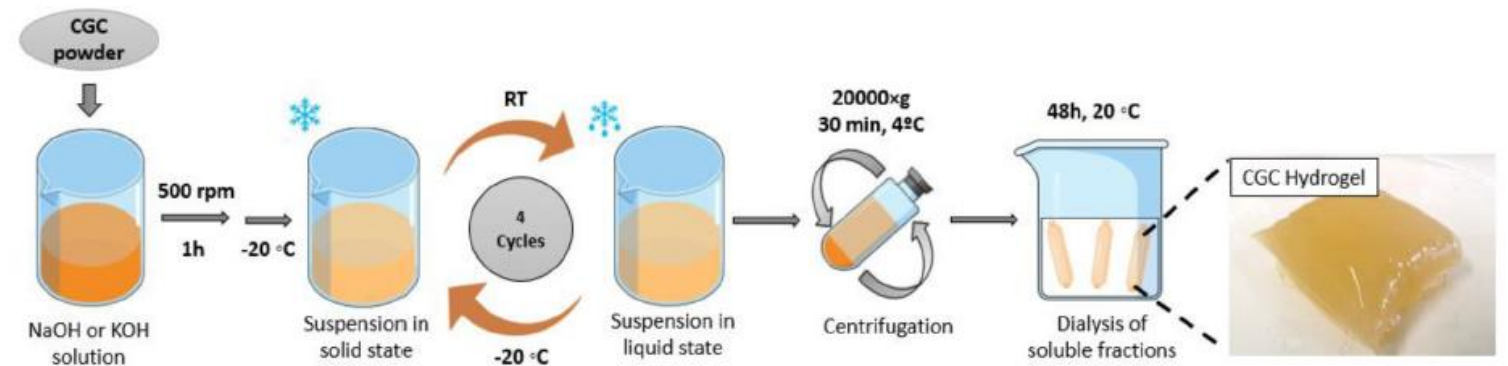

Fig. 1. Schematic illustration of CGC hydrogels preparation, comprising polymer suspension in the alkali systems, application of freeze/thaw cycles for polymer dissolution and dialysis for polymer gelation.

\section{Results and discussion}

\subsection{Hydrogels formation}

Four alkali solvent systems, $\mathrm{NaOH}$ and $\mathrm{KOH}$ solutions, either at $1 \mathrm{~mol} / \mathrm{L}$ or $5 \mathrm{~mol} / \mathrm{L}$, were used for CGC dissolution using a freeze-thaw procedure (Fig. 1), proposed for dissolution of chitin and cellulose $[1,4,8,9]$. At temperatures below the freezing point, the hydrated alkali component disrupts the polymer chain matrix by breaking the interand intramolecular hydrogen bonds. The hydrogels were obtained by dialyzing the CGC solutions, against deionized water until complete removal of the salt ions.

As shown in Fig. 2, the CGC hydrogels presented a yellow coloration and were translucid. The yellow color might be due to the presence of protein whose content in the gels was between 4.4 and $8.8 \mathrm{wt} \%$. The gels' shape was molded by the dialysis tubing, thus showing that the CGC hydrogels obtained by this methodology can be molded into other shapes by inducing gelation in appropriate molds.

As shown in Table 1, no sodium was detected in Na1 and Na5 samples, and only traces of potassium (0.14-0.17 wt\%) were detected in $\mathrm{K} 1$ and $\mathrm{K} 5$ hydrogels. In the alkali solutions, CGC molecules do not undergo gelation due to the strong ionic forces that promoted their solvation. During the dialysis procedure, the salt ions diffuse from the solutions into the dialysis water. The lack of ions available to promote
CGC molecules solvation results in the reduction of the ionic forces and allows more interaction among the CGC molecules, thus inducing gel formation.

The chitin:glucan molar ratios in all hydrogels ranged between 22:78 and 27:73 mol\% (Table 1), which is similar to the composition of the original CGC sample $(29: 71 \mathrm{~mol} \%)$ and also to that reported in previous studies (25:75mol\%) [10]. Although a slight reduction of the chitin fraction in the co-polymer hydrogels was noticed in comparison with the original sample, these results show that CGC was effectively dissolved in the alkali solvents used. All hydrogels presented a water content above $90 \%$ which is characteristic of this type of structures. Nevertheless, hydrogels prepared by dissolution of CGC in $\mathrm{NaOH}$ solutions had a slightly higher polymer content (2.07-2.28 wt\%) than the ones prepared with $\mathrm{KOH}$ (1.42-1.70 wt\%) (Table 1).

\subsection{Morphological characterization}

The morphological characteristics of the CGC hydrogels were assessed by cryo-SEM analysis (Figs. 3 and 4). Fig. 3 shows the Kbased hydrogels microstructure, which consisted of a complex threedimensional network made of CGC polymer chains, holding water inside. Both K-based hydrogels, K1 and K5, exhibited homogenous microstructures (Fig. 3a and c). Upon magnification (Fig. 3b and d), some differences of the pore sizes of K1 and K5 hydrogels can be noticed
(A)
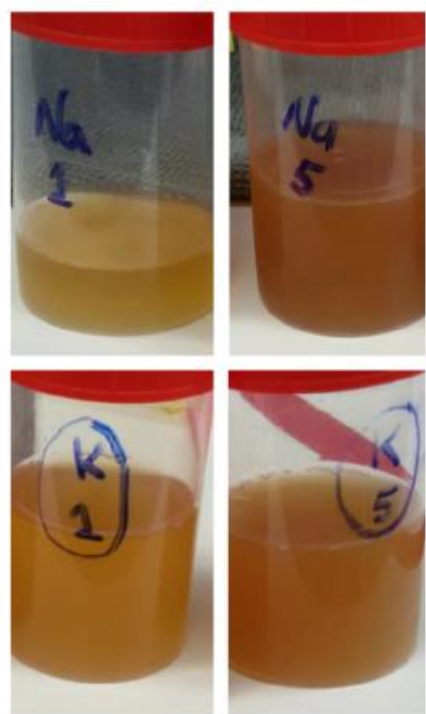

(B)
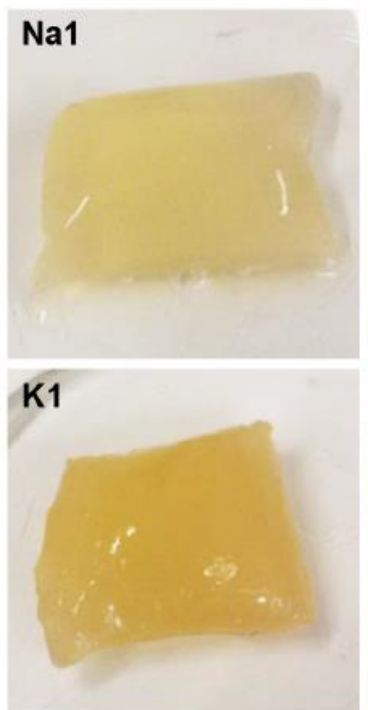

$\mathrm{Na5}$

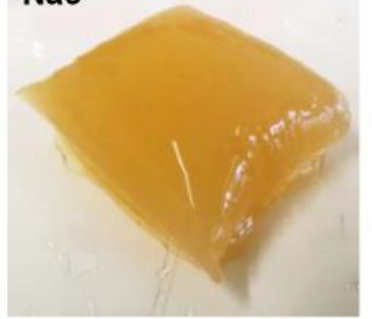

K5

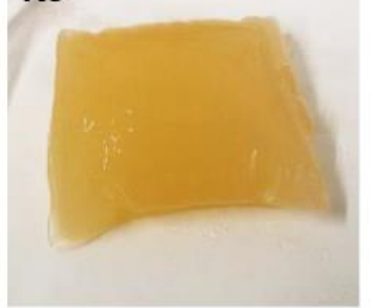

Fig. 2. Photographs of: (A) the CGC solutions obtained by the freeze-thaw procedure in the alkali solvent systems: $\mathrm{NaOH} 1 \mathrm{~mol} / \mathrm{L}$ (Na1 hydrogel) and $5 \mathrm{~mol} / \mathrm{L}$ ( $\mathrm{Na} 5$ hydrogel) and in $\mathrm{KOH}$ $1 \mathrm{~mol} / \mathrm{L}$ (K1 hydrogel) and $5 \mathrm{~mol} / \mathrm{L}$ (K5 hydrogel); (B) the corresponding CGC hydrogels obtained by dialysis of the alkali solutions against deionized water. 
Table 1

Chemical characterization of CGC hydrogels (n.d. - not detected)

\begin{tabular}{llllll}
\hline Sample & $\begin{array}{l}\text { Polymer content } \\
(\text { wt\%) }\end{array}$ & $\begin{array}{l}\text { Water content } \\
\text { (wt\%) }\end{array}$ & $\begin{array}{l}\text { Na content } \\
\text { (wt\%) }\end{array}$ & $\begin{array}{l}\text { K content } \\
\text { (wt\%) }\end{array}$ & $\begin{array}{l}\text { Chitin:glucan ratio } \\
\text { (mol\%) }\end{array}$ \\
\hline CGC & - & - & n.d. & n.d. & $29: 71$ \\
Na1 gel & 2.07 & 97.93 & n.d. & - & $27: 73$ \\
Na5 gel & 2.28 & 97.72 & n.d. & 0.14 & $25: 75$ \\
K1 gel & 1.42 & 98.58 & - & $24: 76$ \\
K5 gel & 1.70 & 98.30 & - & $22: 78$ \\
\hline
\end{tabular}

that suggest the influence of $\mathrm{KOH}$ concentration on the gels' network formation. Hydrogels prepared with $\mathrm{KOH} 1 \mathrm{~mol} / \mathrm{L}$ presented a less compact network with larger pores (Fig. $3 \mathrm{~b}$ ) when compared to the hydrogels prepared with $\mathrm{KOH} 5 \mathrm{~mol} / \mathrm{L}$ (Fig. 3d). A similar microstructure was reported by Nogueira et al. [18] for silk fibroin hydrogels prepared by dissolution in a ternary solvent of $\mathrm{CaCl}_{2}: \mathrm{CH}_{3} \mathrm{CH}_{2} \mathrm{OH}: \mathrm{H}_{2} \mathrm{O}$ followed by dialysis for calcium removal and hydrogel formation [19].

The microstructure of the Na-based hydrogels exhibited a more compact and denser network than the ones prepared with $\mathrm{KOH}$ (Fig. $4 \mathrm{a}$ and c). In addition, they presented heterogeneous and irregular structures. This fact may be explained by the difference in ionic radius of sodium and potassium atoms. During the hydrogelation process, the sodium atoms may have induced the formation of smaller pores within the hydrogel structure, while the bigger potassium atoms may have allowed the formation of larger pores in the polymeric structure. The $\mathrm{NaOH}$ concentration used for CGC dissolution and hydrogel preparation seems to have influenced the microstructure of the formed hydrogels. Na5 CGC hydrogels presented more regions of dense networks (Fig. 4c) than the Na1 CGC hydrogels (Fig. 4a). Upon magnification, small pores with thick pore walls are detected that explain the tighter microstructure of the hydrogels (Fig. 4b and d). The wide area of dense regions of Na5 CGC hydrogels has limited a clear observation of the pore size (Fig. 4d). The porous structure CGC hydrogels present render them potentially suitable for use in applications such as drug delivery vectors and/or as matrices for cells in tissue engineering.

\subsection{Rheological properties}

The mechanical properties of hydrogels are determinant to envisage the functional purposes of these biomaterials. In order to assess those parameters, the viscoelastic properties of CGC hydrogels were studied. As shown in Fig. 5, for the two types of CGC hydrogels prepared with either $\mathrm{NaOH}$ or $\mathrm{KOH}$ as solvent systems, it can be noticed that the storage moduli $\left(G^{\prime}\right)$ exhibited values one order of magnitude higher than the loss moduli $\left(\mathrm{G}^{\prime \prime}\right)$ in the whole range of frequencies. This fact is consistent with the gel-like nature of the samples. Similar profiles were obtained for hydrogels developed using hyaluronic acid (HA) crosslinked with divinyl sulfone [5]. Despite the identical behavior, CGC
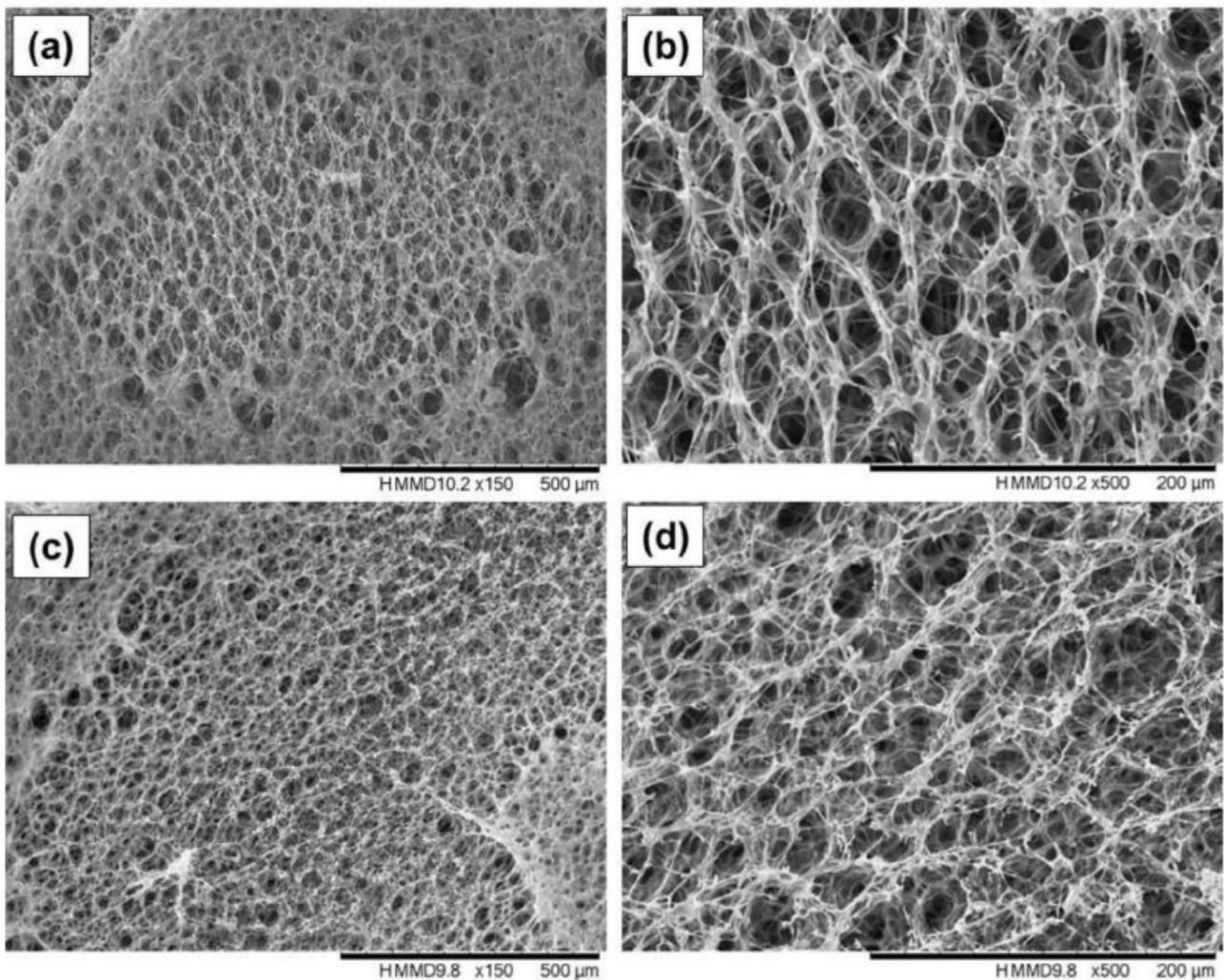

Fig 3. Cryo-SEM images of the $\mathrm{K} 1$ hydrogel prepared with $\mathrm{KOH} 1 \mathrm{~mol} / \mathrm{L}$ (a, b) and of the $\mathrm{K} 5$ hydrogel prepared with $5 \mathrm{~mol} / \mathrm{L}$ (c, d). Hydrogels' regions observed under magnification $500 \times$ (b, d) were expanded from images presented under magnification $150 \times(a, c)$. 

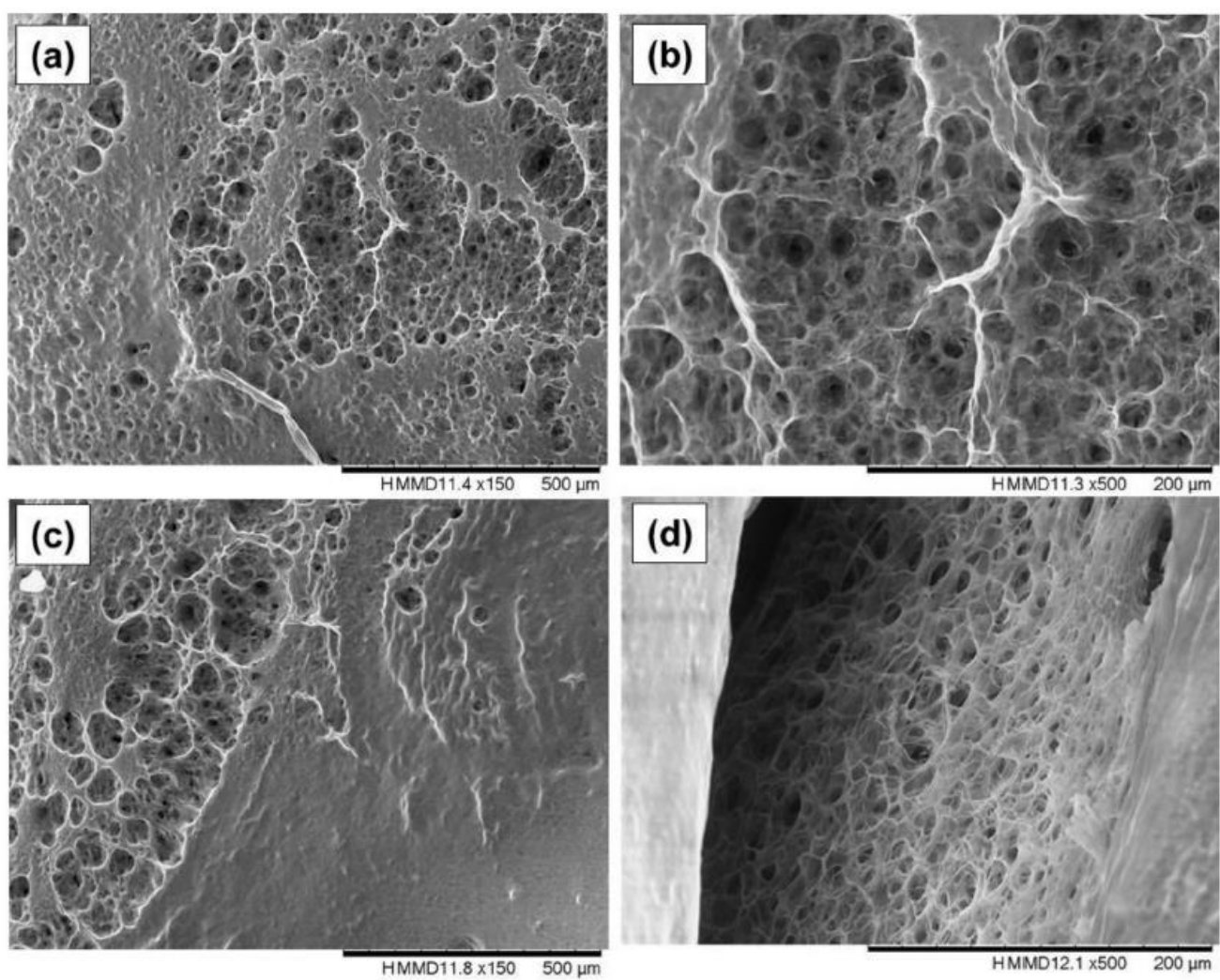

Fig. 4. Cryo-SEM images of the $\mathrm{Na} 1$ hydrogels prepared with $\mathrm{NaOH} 1 \mathrm{~mol} / \mathrm{L}$ (a, b), and of the $\mathrm{Na} 5$ hydrogels prepared 5 mol/L(c, d). Hydrogels' regions observed under magnification $500 \times$ (b, d) were expanded from images presented under magnification $150 \times(a, c)$.

hydrogels revealed to have improved mechanical properties compared to HA hydrogels. In particular, for the same frequency $(0.1 \mathrm{~Hz})$, Na-based CGC hydrogels presented storage moduli values of $389 \mathrm{~Pa}$ and $685 \mathrm{~Pa}$ ( $\mathrm{Na} 5$ and $\mathrm{Na}$ 1, respectively), while for the $\mathrm{HA}$ hydrogels $\mathrm{G}$ lower value was reported ( $304.30 \mathrm{~Pa}$ ). On the other hand, lower storage moduli (218 and $270 \mathrm{~Pa}$ ) were obtained with K1 and K5 CGC hydrogels, respectively. Lower values of $\mathrm{G}^{\prime}$ indicate that K-based CGC hydrogels exhibited lower elastic properties when stress is applied.

As can be observed, CGC hydrogels prepared using $\mathrm{NaOH}$ (Fig. 5a) exhibited higher dynamic moduli than CGC hydrogels prepared with $\mathrm{KOH}$ (Fig. 5b), which is related to stronger mechanical properties. This result is in line with the morphology analysis, in which Na-based CGC
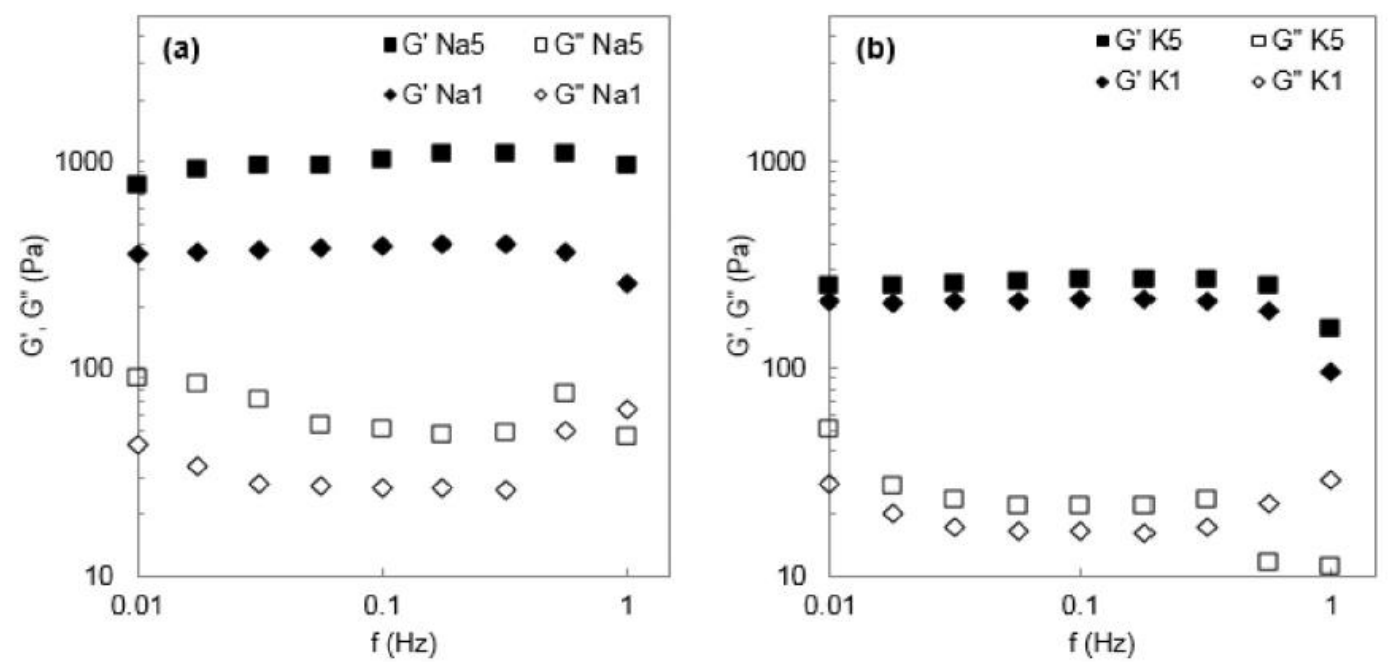

Fig. 5. Rheological properties of CGC hydrogels using (a) $\mathrm{NaOH}$ ( $\mathrm{Na} 1$ and Na5 hydrogels) and (b) $\mathrm{KOH}$ ( $\mathrm{K} 1$ and $\mathrm{K} 5$ hydrogels). Mechanical spectrum storage (G', solid symbols) and loss moduli ( $\mathrm{G}^{\prime \prime}$, open symbols). 
(A)

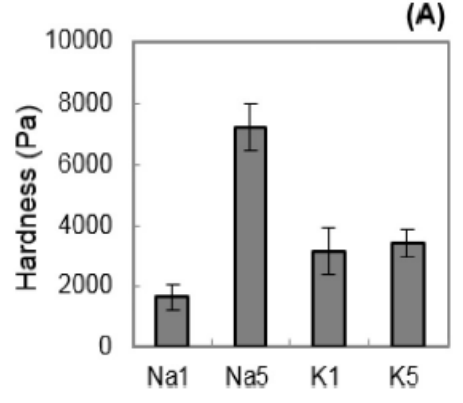

(B)

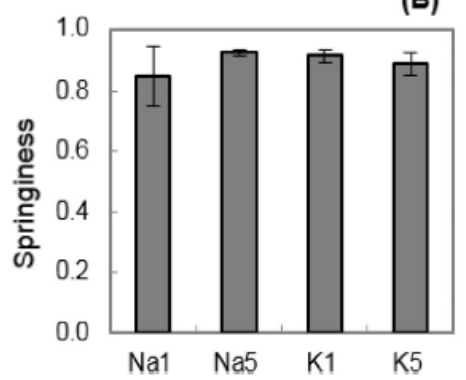

(C)

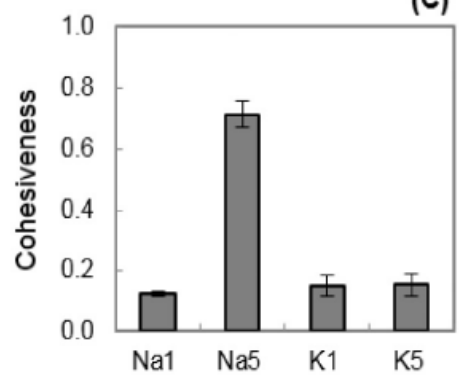

Fig 6. Texture profile analysis parameters for hydrogels prepared with $\mathrm{NaOH} 1 \mathrm{~mol} / \mathrm{L}$ (Na1) and $5 \mathrm{~mol} / \mathrm{L}(\mathrm{Na} 5)$, and with $\mathrm{KOH} 1 \mathrm{~mol} / \mathrm{L}$ (K1) and $5 \mathrm{~mol} / \mathrm{L}$ (K5): (A) hardness, (B) springiness and $(\mathrm{C})$ cohesiveness.

hydrogels exhibited a denser structure with smaller pores with thicker pore walls (Figs. 3 and 4). As mentioned above, the characteristics of CGC hydrogels microstructure may be affected by the difference of ionic radius between sodium and potassium atoms.

Additionally, the mechanical spectra of CGC hydrogels shows that as the concentration of alkali solvents used increased, both dynamic moduli increased indicating a stronger structure (Fig. 5). In fact, higher values of both moduli, $\mathrm{G}^{\prime}$ and $\mathrm{G}^{\prime \prime}$, were observed for the hydrogels prepared with $5 \mathrm{~mol} / \mathrm{L}$ when compared to those for $1 \mathrm{~mol} / \mathrm{L}$, this fact being more evident for Na-based CGC hydrogels. The observed changes may be related with the higher ionic strength present during formation of the Na5 and $\mathrm{K} 5$ hydrogel structures. In fact, it was reported that the ionic strength has a significant impact on hydrogelation and modulation of hydrogels' properties [20]. For example, Martínez-Ruvalcaba and coworkers reported that for the same polymer content in chitosanxanthan hydrogels (7\%), the storage moduli increased around 6 times with the ionic strength of the different dispersion media used, namely distilled water and $0.05 \mathrm{~mol} / \mathrm{L}$ potassium phosphate monobasicsodium hydroxide buffer [21].

The differing properties displayed by the gels obtained under different ionic strength and cations render them potentially suitable for distinct applications: the stiffer structures of the Na-based gels may be used, for example, for the development of tissue scaffolds, while the more elastic K-based gels would be more appropriate for example as wound dressings.

\subsection{Texture profile analysis}

Texture profile analysis (TPA) parameters were obtained by applying a 50\% strain of the hydrogels' original height in a double compression cycle. The results obtained, including hardness, springiness and cohesiveness, are represented in Fig. 6. It may be observed that the hardness, which is related to the maximum force required to cause the selected deformation of the gel [22], was affected by the type of alkali and by the ionic strength of the solvent used to prepare the hydrogels (Fig. 6A). Thus, a considerably higher hardness was obtained for the Na5 hydrogels $(7229 \pm 781 \mathrm{~Pa})$ when compared to Na1 hydrogels ( $1647 \pm 411 \mathrm{~Pa}$ ), showing that a higher ionic strength favored the formation of a stronger gel in the presence of $\mathrm{NaOH}$. On the other hand, for the K-based CGC hydrogels, the hardness values were similar for both ionic strength values tested $(3169 \pm 768$ and $3423 \pm$
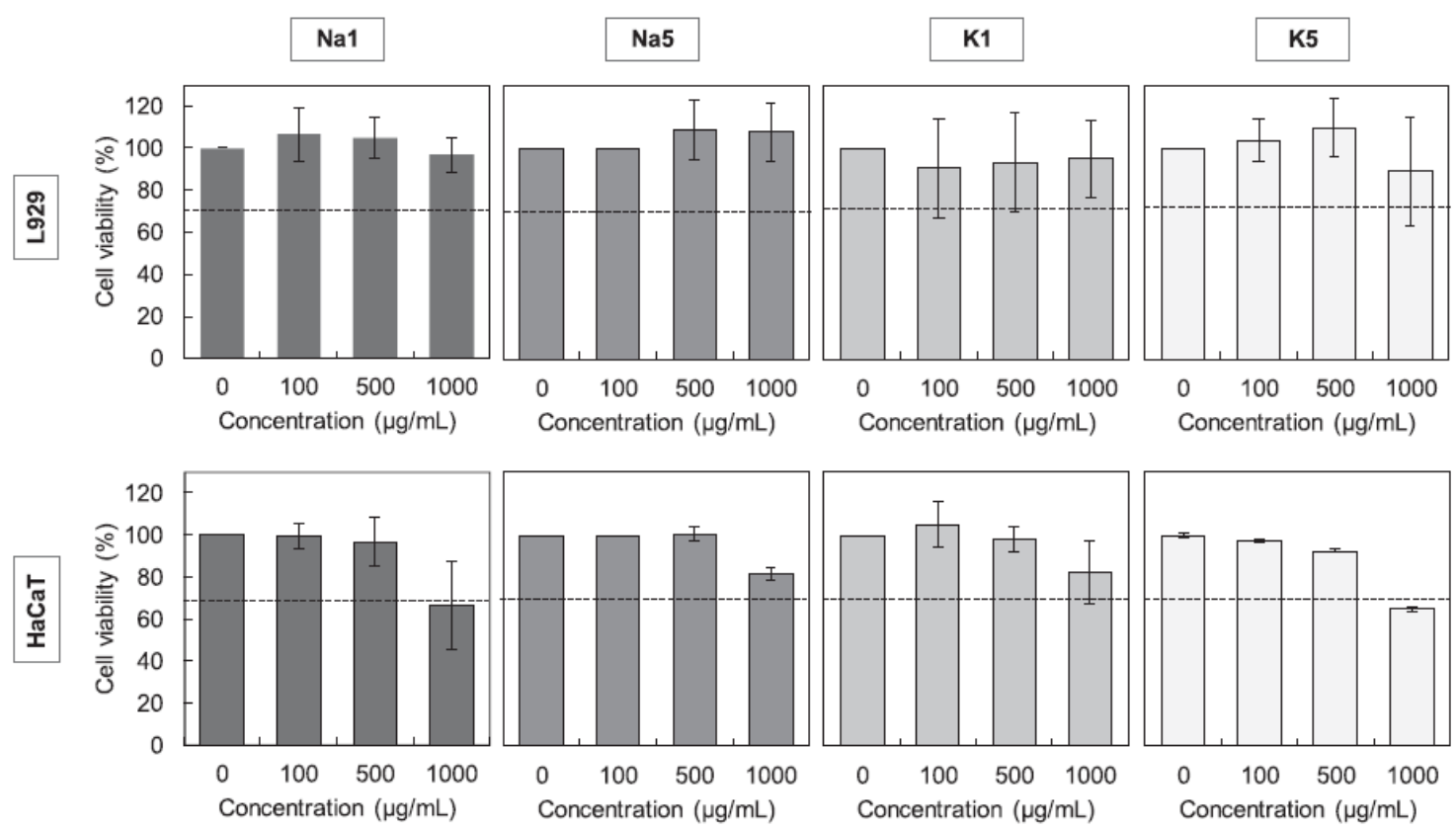

Fig 7. L929 fibroblasts and HaCaT keratinocytes viability when exposed to different concentrations of freeze dried CGC hydrogels dissolved in NaOH $1 \mathrm{~mol} / \mathrm{L}$, for $24 \mathrm{~h}$. The dotted line at $70 \%$ represents the viability limits for non-toxic condition (ISO 10993-5, 2009). 
$450 \mathrm{~Pa}$, respectively). Moreover, the hardness of both K-based hydrogels was lower than that of the Na5 hydrogel (Fig. 6A).

Springiness reflects how well the gel sample physically springs back after it has been deformed during the first compression and has been allowed to wait for a period between compressions [22]. It can be observed that similar values of springiness were obtained for all CGC hydrogels (Fig. 6B), which is indicative of an elastic behavior, as the fraction of the sample's original height is quite high, in a range of 0.85 to 0.93 . Cohesiveness is related to how well the product withstands compression, so that, if a hard product maintains its internal structure without failures when subjected to compression, it would also present a high cohesiveness [22]. This fact was only substantially observed for Na5 hydrogels, presenting a value of $0.716 \pm 0.043$ (Fig. 6C), while the other hydrogels, where much less cohesive. The results suggest that the energy required for the second compression was significantly lower than that for the first compression, indicating that the gel matrix of the $\mathrm{Na} 1$ and $\mathrm{K}$-hydrogels was disrupted during the first compression. Low strength of polymer chain molecular interactions may have led to this hydrogel behavior [2].

Concomitantly with the mechanical spectra (Fig. 5), the TPA results demonstrate that the ionic strength of the solvent used has more impact on Na-based hydrogels than on K-based hydrogels (Fig. 6). Additionally, the Na5 hydrogels presented improved mechanical properties since significantly higher hardness and cohesiveness values were observed.

Several authors reported the improvement of the texture properties of hydrogels by increasing the concentration of the polymer $[23,24]$. The same strategy could be used to improve mechanical properties of CGC hydrogels.

\subsection{Biocompatibility of the CGC hydrogels}

The evaluation of CGC hydrogels' biocompatibility was performed using L929 fibroblasts (International Standard ISO 10993-5:2009(E)). Fig. 7 shows the results of the cytotoxicity tests on the CGC hydrogels. The cell viability values on all tested hydrogels are greater than $90 \%$, indicating nearly no cytotoxicity to the fibroblasts (Fig. 7A). The noncytotoxicity of the solvent used to prepare the CGC samples $(\mathrm{NaOH}$ $1 \mathrm{~mol} / \mathrm{L}$ ) was confirmed by exposing the cells solely to this solvent (Fig. SM-1). Chang and collaborators [4] have reported similar biocompatibility results for chitin-based hydrogels towards $293 \mathrm{~T}$ cells, most probably related to the biological nature of these macromolecules. Similarly, Abdel-Mohsen et al. [16] have demonstrated that chitin/ chitosan-glucan complex (ChCsGC) revealed to be non-toxic for mouse fibroblasts cells (NIH-3T3) over the all concentrations tested $(100-1000 \mu \mathrm{g} / \mathrm{mL})$.

To further evaluate the biocompatibility of the CGC hydrogels for skin applications, HaCaT keratinocytes cells were used to assess the hydrogels for $24 \mathrm{~h}$. Fig. 7B shows the viability of keratinocytes. Compared with the control group, the HaCaT cells exposed to the highest CGC hydrogels concentration $(1000 \mu \mathrm{g} / \mathrm{mL})$ exhibited reduced cellular viability to values around $70 \%$. These results confirm that the CGC hydrogels could be used as an excellent matrix having biocompatibilities, with potential applications in the field of skin wound and skin therapies. Biocompatibility of chitin-based hydrogels obtained by various methods has been reported in the literature through in vitro cytotoxicity tests [25-27].

\section{Conclusions}

This study reported for the first time the preparation of hydrogels based on the yeast biopolymer CGC. It was demonstrated that CGC gels form spontaneously during dialysis of CGC alkali solutions, obtained by the freeze-thaw procedure. The mechanical properties of the CGC hydrogels can be tailored according to the alkali solvent used for CGC dissolution, either $\mathrm{NaOH}$ or $\mathrm{KOH}$, and also by changing the solutions ionic strength. Moreover, the best performing hydrogels in terms of mechanical properties, namely, higher hardness and cohesiveness, were the ones obtained with $\mathrm{NaOH} 5 \mathrm{~mol} / \mathrm{L}$. Due to their biocompatibility, CGC hydrogels are promising materials for use in in several biomedical areas. Their characteristics, namely, their porous structure and mechanical properties, supports their potential use as drug delivery vectors and/or as matrices for cells in tissue engineering.

Supplementary data to this article can be found online at https://doi. org/10.1016/j.ijbiomac.2019.11.141.

\section{Author contributions}

The manuscript was written through contributions of all authors. All authors have given approval to the final version of the manuscript.

\section{Funding}

This project was supported by the Unidade de Ciências Biomoleculares Aplicadas (UCIBIO), the Associated Laboratory for Sustainable Chemistry - Clean Processes and Technologies (LAQV), and Linking Landscape, Environment, Agriculture and Food (LEAF), which are financed by national funds from FCT-MCTES (UID/Multi/04378/ 2013, UID/QUI/50006/2013, PEst-OE/AGR/UI0245/2014, UID/QUI/ 50006/2019) and co-financed by the ERDF, under the PT2020 Partnership Agreement (POCI-01-0145-FEDER-007728, POCI-01-0145FEDER-007265). FCT-MCTES is also acknowledged for funding project CEECIND/01620/2017 and for grant BD/140829/2018.

\section{References}

[1] X. Shen, J.L. Shamshina, P. Berton, G. Gurauc, R.D. Rogers, Hydrogels based on cellulose and chitin: fabrication, properties, and applications, Green Chem. 18 (2016) 53 https://doi.org/10.1039/c5gc02396c.

[2] G. Calixto, A.C. Yoshii, H. Rocha e Silva, B.S.F. Cury, M. Chorilli, Polyacrylic acid polymers hydrogels intended to topical drug delivery: preparation and characterization, Pharm. Dev. Technol. 20 (4) (2015) 490-496, https://doi.org/10.3109/10837450. 2014.882941.

[3] H. Tang, H. Chen, B. Duan, A. Lu, L. Zhang, Swelling behaviors of superabsorbent chitin/carboxymethylcellulose hydrogels, J. Mater. Sci. 49 (2014) 2235-2242, https:// doi.org/10.1007/s 10853-013-7918-0.

[4] C. Chang, S. Chen, L. Zhang, Novel hydrogels prepared via direct dissolution of chitin at low temperature: structure and biocompatibility, J. Mater. Chem. 21 (2011) 3865-3871, https://doi.org/10.1039/C0JM03075A.

[5] A. Borzacchiello, L. Russo, B.M. Malle, K. Schwach-Abdellaoui, L. Ambrosio, Hyaluronic acid based hydrogels for regenerative medicine applications, Biomed. Res. Int. 871218 (2015) https://doi.org/10.1155/2015/871218.

[6] X. Shen, J.J. Shamshina, P. Berton, J. Bandomir, H. Wang, G. Gurau, R.D. Rogers, Comparison of hydrogels prepared with ionic-liquid-isolated vs commercial chitin and cellulose, ACS Sustain. Chem. Eng. 4 (2016) 471-480, https://doi.org/10.1021/ acssuschemeng. 5 b01400.

[7] C. Mukesh, D. Mondal, M. Sharma, K. Prasad, Choline chloride-thiourea, a deep eutectic solvent for the production of chitin nanofibers, Carbohydr. Pol. 103 (2014) 466-471, https://doi.org/10.1016/j.carbpol 2013.12.082.

[8] Y. Fang, B. Duan, A. Lu, M. Liu, H. Liu, X. Xu, L Zhang Intermolecular interaction and the extended wormlike chain conformation of chitin in $\mathrm{NaOH} / \mathrm{urea}$ aqueous solution, Biomacromolecules 16 (2015) 1410-1417, https://doi.org/10.1021/acs. biomac.5b00195.

[9] X. Hu, Y. Tang, Q. Wang, Y. Li, J. Yang, Y. Dub, J.F. Kennedy, Rheological behaviour of chitin in NaOH/urea aqueous solution, Carbohydr. Pol. 83 (2011) 1128-1133, https://doi.org/10.1016/j.carbpol.2010.09.014.

[10] L. Farinha, P. Duarte, A. Pimentel, E. Plotnikova, B. Chagas, L. Mafra, C. Grandfils, F. Freitas, E. Fortunato, M.A.M. Reis, Chitin-glucan complex production by Komagataella pastoris: downstream optimization and product characterization, Carbohydr. Pol. 130 (2015) 455-464, https://doi.org/10.1016/j.carbpol.2015.05.034

[11] A.V. Pestov, S.V. Drachuk, O.V. Koryakova, Y.G. Yatluk, Isolation and characterization of chitin-glucan complexes from the mycothallus of fungi belonging to Russula genus, Chem. Sust. Dev. 17 (2009) 281-287.

[12] C. Roca, B. Chagas, I. Farinha, F. Freitas, L. Mafra, F. Aguiar, R. Oliveira, M.A.M. Reis, Production of yeast chitin-glucan complex from biodiesel industry byproduct, Process Biochem. 47 (11) (2012) 1670-1675, https://doi.org/10.1016/j.procbio.2012 04.004.

[13] A.M. Neyrinck, S. Possemiers, W. Verstraete, F. De Backer, P.D. Cani, N.M. Delzenne, Dietary modulation of clostridial cluster XIVa gut bacteria (Roseburia spp.) by chitin-glucan fiber improves host metabolic alterations induced by high-fat diet in mice, J. Nutr. Biochem. 23 (1) (2012) 51-59, https://doi.org/10.1016/j.jnutbio. mice, J. Nutr.
2010.10 .008 
[14] S. Gautier, E. Xhauflaire-Uhoda, P. Gonry, G.E. Pierard, Chitin-glucan, a natural cell scaffold for skin moisturization and rejuvenation, Int. J. Cosmetic Sci. 30 (6) (2008) 459-469, https://doi.org/10.1111/j.1468-2494.2008.00470.x.

[15] A. Bornet, P.L. Teissedre, Chitosan, chitin-glucan and chitin effects on minerals (iron, lead, cadmium) and organic (ochratoxin A) contaminants in wines, Eur. Food Res. Technol. 226 (4) (2008) 681-689.

[16] A.M. Abdel-Mohsen, J. Jancar, D. Massoud, Z. Fohlerova, H. Elhadidy, Z. Spotz, A. Hebeish, Novel chitin/chitosan-glucan wound dressing: isolation, characterization, antibacterial activity and wound healing properties, Int. J. Pharm. 510 (2016) 86-99, https://doi.org/10.1016/j.jpharm 2016.06.003.

[17] D. Araú́jo, F. Freitas, C. Sevrin, C. Grandfils, M.A.M. Reis, Co-production of chitinglucan complex and xylitol by Komagataella pastoris using glucose and xylose mixtures as carbon source, Carbohydr. Pol. 166 (2017) 24-30, https://doi.org/10.1016/J. carbpol.2017.02.088.

[18] N.R. Raia, B.P. Partlow, M. McGill, E.P. Kimmerling, C.E. Ghezzi, D.L. Kaplan, Enzymatically crosslinked silk-hyaluronic acid hydrogels, Biomaterials 131 (2017) 58-67, https://doi.org/10.1016/jbiomaterials.2017.03.046

[19] G.M. Nogueira, M.A. Moraes, A.C.D. Rodas, OZ. Higa, M.M. Beppu, Hydrogels from silk fibroin metastable solution: formation and characterization from a biomateria perspective, Mat. Sci. Eng. C 31 (5) (2011) 991-1001, https://doi.org/10.1016/j. msec.2011.02019.

[20] Y. Feng, M. Taraban, B. Yu, The effect of ionic strength on the mechanical, structura and transport properties of peptide hydrogels, Soft Matter 8 (46) (2012) 11723-11731, https://doi.org/10.1039/C2SM26572A.
[21] A. Martínez-Ruvalcaba, E. Chomet, D. Rodrigue, Viscoelastic properties of dispersed chitosan/xanthan hydrogels, Carbohydr. Pol. 67 (4) (2007) 586-595, https://doi. org/10.1016/j.carbpol.2006.06.033.

[22] G.R. Sanderson, Gellan gum, in: P. Harris (Ed.), Food Gels, Elsevier Science Publishing Co., Inc, New York 1990, pp. 201-232.

[23] M.H. Lau, J. Tang, A.T. Paulson, Texture profile and turbidity of gellan/gelatin mixed gels, Food Res. Int. 33 (8) (2000) 665-671, https://doi.org/10.1016/S0963-9969(00) 00111-3.

[24] J.Hurler, A. Engesland, B.P. Kermany, N. Skalko-Basnet, Improved texture analysis for hydrogel characterization: gel cohesiveness, adhesiveness, and hardness, J. Appl. Pol. Sci. 125 (1) (2012) 180-188, https://doi.org/10.1002/app.35414.

[25] J. Duan, X. Liang, Y. Cao, S. Wang, L. Zhang, High strength chitosan hydrogels with biocompatibility via new avenue based on constructing nanofibrous architecture, Macromol 48 (8) (2015) 2706-2714, https://doi.org/10.1021/acs.macromol. 5 b00117.

[26] B. Tyliszczak, A. Drabczyk, S. Kudłacik, Comparison of hydrogels based on commercial chitosan and Beetosan@ containing nanosilver, Molecules 22 (2017) 61, https:// doi.org/10.3390/molecules22010061,

[27] L. Zhao, L. Niu, H. Liang, H. Tan, C. Liu, F. Zhu, pH and glucose dual-responsive injectable hydrogels with insulin and fibroblasts as bioactive dressings for diabetic wound healing, ACS Appl. Mater. Interfaces 9 (2017) 37563-37574, https://doi.org/10. 1021/acsami.7b09395. 